\title{
Determinação do coeficiente de reaeração em rios através do uso do traçador gasoso GLP
}

\section{Determination of stream reaeration coefficients by use of GLP gas tracer}

\author{
Adilson Pinheiro \\ Engenheiro civil. Doutor em Física e Química do Ambiente. Professor do Departamento de Engenharia Civil da Fundação Universidade Regional de \\ Blumenau (FURB) - Blumenau (SC), Brasil.
}

\section{Guilherme Faht}

Químico. Mestre em Engenharia Ambiental pela Fundação Universidade Regional de Blumenau (FURB) - Blumenau (SC), Brasil.

\section{Marcos Rivail da Silva}

Químico. Doutor em Química Ambiental pela Université de Bordeaux - Bordeaux, França. Professor do Departamento de Química da Fundação Universidade Regional de Blumenau (FURB) - Blumenau (SC), Brasil.

\section{Resumo}

A oxigenação da água constitui-se em importante fator no desenvolvimento do fenômeno de autodepuração de rios. A reposição do oxigênio dissolvido pode ser representada pelo coeficiente de reaeração $\left(\mathrm{K}_{2}\right)$. Neste trabalho foi testado o uso do GLP como fonte do traçador gás propano em ensaios de reaeração. Foram executados 15 ensaios em trechos de rios com características hidrodinâmicas distintas. Os experimentos mostraram que a utilização do GLP como fonte do traçador gasoso é viável, tanto do ponto de vista analítico, quanto do ponto de vista econômico. Do ponto de vista analítico, os resultados foram equiparáveis e até superiores à técnica tradicional de traçador gasoso. Do ponto de vista econômico, houve redução do custo para a realização de cada ensaio.

Palavras-chave: coeficiente de reaeração; traçador gasoso; propano.

\begin{abstract}
The water oxygenation constitutes an important factor in the development of self-purification phenomenon in rivers. The replenishment of dissolved oxygen in water can be represented by the reaeration coefficient $\left(\mathrm{K}_{2}\right)$. This work was used propane contained in the GLP as tracer in reaeration tests. The tests showed that the use of LPG is feasible, both from analytical point of view, as of the economic. From an analytical standpoint, the results were comparable and even superior to the traditional technique of tracer gas. From an economic standpoint, the cost to perform each test has been reduced.
\end{abstract}

Keywords: reaeration coefficient; tracer gas; propane.

\section{Introdução}

Os processos de oxigenação são de suma importância à garantia da vida em um corpo de água. A oxigenação pode ser proveniente dos seres fotossintetizantes situados no meio aquático, assim como da atmosfera, sendo esse último dominante no sistema aquático.

A reposição do oxigênio é expressa através de um parâmetro nomeado de coeficiente de reaeração $\left(\mathrm{K}_{2}\right)$. Através dele é possível quantificar o processo de transferência de oxigênio da atmosfera para a massa líquida, parâmetro essencial para operação de modelos de qualidade de água (AMBROSE; WOOL; BARNWELL, 2009; FAN; KO; WANG, 2009). Esses modelos são empregados na avaliação da capacidade de autodepuração natural de rios.
O coeficiente de reaeração pode ser determinado através de modelos de natureza teórica, empírica e semiempírica, além de equipamentos e métodos desenvolvidos exclusivamente para essa finalidade. Destaca-se que os modelos teóricos geralmente apresentam inconvenientes, pois necessitam de parâmetros não facilmente relacionados às características físicas e hidráulicas do corpo de água. Os modelos empíricos e semiempíricos satisfazem somente o local ao qual eles foram originados. Devido à singularidade das características físicas e hidráulicas, para melhor compreensão e precisão de um estudo, é necessária a determinação do coeficiente de reaeração $\left(\mathrm{K}_{2}\right)$ para cada curso de água.

As técnicas aceitas para a determinação do $\mathrm{K}_{2}$, segundo Cox (2003), são: a técnica do balanço de oxigênio dissolvido, desenvolvida por Streeter e Phelps (1925), a técnica da perturbação do equilíbrio, 
desenvolvida por Gameson, Truesdale e Downing (1955) e a técnica dos traçadores, desenvolvida por Tsivoglou e Wallace (1972). Após muitos estudos, verificou-se que a técnica dos traçadores gasosos tem se mostrado o método mais eficiente na determinação do K (RATHBUN; GRAND, 1978; YOTSUKURA et al., 1983; YOTSUKURA; STEDFAST; JIRKA, 1984; PARKER; GAY, 1987; LICO; TAYLOR, 2000).

$\mathrm{Na}$ sua primeira aplicação, a técnica dos traçadores gasosos utilizava gases radioativos, como por exemplo, o criptônio (isótopo - 85). A quantidade de radiação sempre foi considerada o grande inconveniente para a execução dessa técnica, principalmente ao utilizá-la para cursos de água com grandes vazões. Visando melhorar a técnica e expandir sua aplicabilidade, modificações foram realizadas na metodologia, que passou a descartá-los (KILPATRICK et al., 1989; GLEIZER, 1992). Os gases radioativos foram substituídos por hidrocarbonetos leves, dentre eles o etileno e o propano.

A substituição dos gases radioativos reduziu a insalubridade e possibilitou a expansão do uso da técnica. Infelizmente, no Brasil, esses gases (propano e etileno puros) possuem um custo muito elevado que limita a realização de ensaios de reaeração. Poucos trabalhos têm sido desenvolvidos visando a determinação de $\mathrm{K}_{2}$ em rios (GLEIZER, 1992; BARBOSA JUNIOR, 1997; FORMENTINI, 2010) visto que o custo de cada ensaio é elevado. Assim, visando superar a limitação de custo financeiro foi desenvolvida uma modificação na técnica dos traçadores gasosos.

Assim, o objetivo desse trabalho foi avaliar a aplicação de uma técnica confiável, que possibilite maior escala de utilização por apresentar menor custo e maior disponibilidade de material. Testou-se o gás liquefeito de petróleo (GLP) como fonte do traçador gasoso propano, em substituição ao etileno e ao propano puros (grau de pureza superior a 99\%).

\section{Materiais e métodos}

A determinação do $\mathrm{K}_{2}$ através do traçador gasoso GLP envolve quatro etapas de preparação. Primeiramente preocupou-se com a escolha dos traçadores. Após isso, montou-se o sistema de reaeração composto por uma mangueira especial para GLP, um regulador de pressão, um fluxômetro e um difusor. Em seguida, preparou-se a instrumentação analítica para a determinação dos traçadores. Terminada essa etapa, preocupou-se com as condições de campo, como determinação dos pontos de amostragem, representatividade dos locais de coleta, comprimento de mistura e a vazão. Para o desenvolvimento da presente técnica foram executados 15 ensaios perfazendo 450 amostras de traçador conservativo e 75 amostras de traçador gasoso.

\section{Traçadores}

A técnica utiliza dois tipos de traçadores. Um denominado de traçador gasoso (não conservativo no sistema aquático) e outro traçador sólido solúvel (considerado conservativo para o intervalo de tempo experimental).

A mistura de hidrocarbonetos vendida no Brasil como GLP é composta por 40 a $60 \%$ de propano (v/v), 40 a $60 \%$ de butano (v/v), máximo de $11 \%$ de etano e outros gases mais leves (v/v) e 0,5 a 2\% de pentano e outros hidrocarbonetos (v/v) (LIQUIGÁS, 2011).

Dessa mistura de hidrocarbonetos, somente o propano foi utilizado como traçador gasoso. Esse gás possibilita simular o comportamento do oxigênio dissolvido nos cursos de água. Devido a sua baixa reatividade, foi muito utilizado em outras técnicas e trabalhos envolvendo ensaios de reaeração (LICO; TAYLOR, 2000; BARBOSA JUNIOR, 1997; GLEIZER, 1992; KILPATRICK et al., 1989; PARKER; GAY, 1987; YOTSUKURA; STEDFAST; JIRKA, 1984; YOTSUKURA et al., 1983; RATHBUN; GRAND, 1978).

Para a execução dos experimentos foram utilizados botijões do tipo P13. Esse botijão é amplamente utilizado nas instalações residenciais, e ganhou o nome de gás de cozinha porque, segundo a legislação brasileira, ele só deve ser utilizado em residências. Nesse botijão há $13 \mathrm{~kg}$ de GLP, e devido às suas dimensões, pode ser facilmente transportado, inclusive para regiões de difícil acesso. Isso é uma grande vantagem, pois o seu transporte não limita os estudos.

Além do traçador gasoso, utiliza-se um traçador conservativo. Esse traçador serve para controlar o experimento, pois através dele é possível determinar os tempos de viagem e o comprimento de mistura completa. Como traçador conservativo, utilizou-se a substância fluorescente rodamina WT. A rodamina é uma substância de cor vermelha, não tóxica e não carcinogênica. Sua fórmula molecular é $\mathrm{C}_{29} \mathrm{H}_{29} \mathrm{ClN}_{2} \mathrm{Na}_{2} \mathrm{O}_{5}$ e sua massa molar é de $566,9835 \mathrm{~g} \cdot \mathrm{mol}^{-1}$. O fato de ser uma substância facilmente detectável instrumentalmente em baixas concentrações e apresentar pouca reatividade a tornaram um traçador muito utilizado (USEPA, 1989).

\section{Seleção dos trechos experimentais}

Esta é uma das etapas mais importantes da experimentação. A escolha dos trechos determinará a representatividade dos resultados, o tempo de experimentação, o recurso humano necessário e o material gasto. A representatividade dos resultados está ligada à escolha de trechos condizentes às características físicas e hidráulicas do corpo de água. A seleção dos trechos é possível através da análise de mapas e dados hidrológicos. O tempo de experimentação, o recurso humano e o material gasto impactarão no custo do experimento.

Para a execução de um experimento é necessário, no mínimo, a seleção de três seções (o que gera dois trechos). A primeira seção é o local onde serão lançados os traçadores (tanto o conservativo, quanto o gasoso). Ao longo do corpo de água deverá haver mistura completa dos traçadores. Por esse motivo, a segunda seção deverá estar logo após a mistura completa. É na 
segunda seção que ocorrerão as primeiras coletas. O coeficiente de reaeração será determinado pelo trecho formado pela segunda e terceira seção.

A distância entre a primeira e segunda seção foi estimada pelo modelo de comprimento de mistura proposto por Fischer et al. (1979), expresso pelas Equações 1 e 2.

$L_{F}=0,1 \cdot \frac{U \cdot B^{2}}{D_{L}}$

$D_{L}=\frac{0,011 \cdot U^{2} \cdot B^{2}}{H \cdot u}$, o coeficiente de dispersão longitudinal $\left(\mathrm{m}^{2} \cdot \mathrm{s}^{-1}\right)$

Sendo:

$L_{\mathrm{F}}$ : comprimento de mistura lateral $(\mathrm{m})$,

$U$ : velocidade média do trecho $\left(\mathrm{m}^{2} \cdot \mathrm{s}^{-1}\right)$;

$B$ : largura média do trecho $(\mathrm{m})$;

H: declividade $\left(\mathrm{m} \cdot \mathrm{m}^{-1}\right)$;

u: atrito $\left(\mathrm{m}^{2} \cdot \mathrm{s}^{-2}\right)$

Considerou-se a distância calculada pelo modelo de Fischer et al. (1979) como a menor distância entre as duas seções. No entanto, para garantia de mistura completa recomenda-se utilizar uma distância maior (KILPATRICK et al., 1989).

\section{Lançamento do traçador conservativo}

Preparou-se uma solução 20\% (massa/massa) através da dissolução de rodamina WT em água. Fez-se o lançamento instantâneo da solução no ponto central da seção transversal do curso de água.

O volume de solução utilizada depende de informações hidrodinâmicas determinadas durante o ensaio e do limite de quantificação do espectrofluorímetro ou do fluorímetro utilizado. O modelo proposto por Kilpatrick e Cobb (1985) possibilita determinar o volume da solução de rodamina WT a ser lançada instantaneamente. O volume é determinado pela Equação 3.

$V_{s}=\Phi\left(\frac{Q . L}{U}\right)^{\Gamma} C_{p}$

Onde:

$V_{\varsigma}$ : volume da solução de rodamina wt $(m L)$;

$\Phi$ : coeficiente empírico adimensional (usou-se 1,338 x 10-3);

Q: vazão do curso de água $\left(\mathrm{m}^{3} \cdot \mathrm{s}^{-1}\right)$;

$L$ : distância entre a seção de lançamento e a última seção de coleta $(m)$;

U:velocidade média do escoamento $\left(\mathrm{m} \cdot \mathrm{s}^{-1}\right)$;

$C_{p}$ : pico de concentração desejada $\left(\mu g . L^{-1}\right)$;

$\Gamma$ : coeficiente empírico adimensional (usou-se 1)

Uma vez lançada a solução contendo o traçador conservativo, essa se dispersará e percorrerá o curso de água. Mas antes disso foi necessário programar as coletas para monitorar a passagem da nuvem. Isso só se torna possível através do cálculo do tempo de viagem do pico de concentração de rodamina WT. Esse tempo de viagem foi determinado para cada seção de coleta. Os modelos propostos por Boning (1973) possibilitam de acordo a declividade do rio, o cálculo da velocidade de deslocamento do pico de concentração da nuvem do traçador. Eles são expressos pelas Equações 4 e 5 .

- para declividade variando de 0,00012 a 0,0057 m.m.1.

$V_{p}=\left[0,38 \cdot(35,3147 \cdot 2)^{0,40} \cdot \delta^{0,20}\right] \cdot 0,3048$

- para declividade variando de 0,00016 a 0,0023 m.m.

$V_{p}=\left[2,69 \cdot(35,3147 \cdot 2)^{0,26} \cdot \delta^{0,28}\right] \cdot 0,3048$

Onde:

$V_{p}$ : velocidade de deslocamento do pico de concentração $\left(\mathrm{m} . \mathrm{s}^{-1}\right)$;

Q: vazão média do trecho $\left(\mathrm{m}^{3} \cdot \mathrm{s}^{-1}\right)$;

$\delta$ : declividade $\left(\mathrm{m} \cdot \mathrm{m}^{-1}\right)$.

Calculou-se o tempo de viagem do pico entre as duas seções extremas através da equação da velocidade média reajustada (Equação 6).

$T_{p}=\left(\frac{\Delta x}{V_{p}}\right) \cdot 60$

Onde:

$T_{p}$ : tempo de viagem do pico (min);

$\Delta x$ : distância entre os pontos extremos da seção (m);

$V_{p}$ : velocidade de deslocamento do pico de concentração $\left(\mathrm{m} . \mathrm{s}^{-1}\right)$.

A amostragem é um dos aspectos mais críticos, pois ela deve ocorrer de tal forma que os dados sejam representativos dos processos simulados, principalmente relacionados à ponta da nuvem do corante traçador, pois essa avança mais rapidamente que o pico. A programação da amostragem é feita com base na estimativa da velocidade média, que se aplica mais ao pico de concentração do que à ponta da nuvem. Por esse motivo, utilizou-se a abordagem de Taylor.

Taylor, James Junior e Helinsky (1984) analisaram centenas de conjuntos de dados de passagem de nuvens do corante e estabeleceram que a curva de resposta produzida pode ser representada por um triângulo escaleno. Desse triângulo escaleno, um terço da duração do tempo total de passagem foi da ponta da nuvem até o pico, os outros dois terços foi o tempo necessário para a concentração atingir $10 \%$ da concentração do pico. Considerando o trabalho de Taylor, James Junior e Helinsky (1984), é possível estimar o tempo de chegada da ponta da nuvem de traçador conservativo. O tempo de chegada da ponta é o que define o início da amostragem. Considerando que a distância da ponta até o pico da concentração de traçador equivale a um terço do tempo de passagem da nuvem até que a concentração atinja 10\% da concentração do pico, foi estabelecida a expressão (Equação 7). 
$T_{b}=T_{p}-0,23 \cdot T_{p}^{0,86}$

Onde:

$T_{b}$ : tempo para início da amostragem (min);

$\mathrm{T}_{\mathrm{p}}$ : tempo de viagem do pico (min).

Coletaram-se 30 amostras para cada seção de amostragem, pois segundo Kilpatrick et al. (1989), esta quantidade é suficiente para gerar uma curva bem definida. A frequência de coleta foi determinada pela abordagem de Taylor, James Junior e Helinsky (1984), a qual estimou o tempo de residência do traçador. Dividiu-se 30 pelo tempo de residência e obteve-se a frequência de amostragem. A frequência de amostragem pode ser calculada segundo o modelo apresentado na Equação 8.

$F=\frac{30}{3,1 \cdot T_{p}^{0,67}}$

Onde:

F: frequência de amostragem (amostras. $\mathrm{min}^{-1}$ );

$T_{p}$ : tempo de viagem do pico (min).

As amostras foram recolhidas em frascos de polietileno de $100 \mathrm{~mL}$. A coleta da amostra foi feita mergulhando-se o frasco a $30 \mathrm{~cm}$ da superfície do curso de água. Terminada a coleta, o material foi logo em seguida analisado para reduzir a possibilidade de perdas da rodamina WT por efeito de fotodecomposição (BARBOSA JUNIOR, 1997).

Em campo, foram determinadas as concentrações da rodamina WT. As análises foram efetuadas utilizando-se um fluorímetro AquaFluor (Tuner Designs) previamente calibrado. O equipamento proporcionou leitura direta e instantânea da concentração de rodamina WT através da fluorescência. A detecção é feita através de um fotodiodo com faixa de trabalho de 300 a $1.000 \mathrm{~nm}$. O limite de detecção foi de $0,4 \mu \mathrm{g} \cdot \mathrm{L}^{-1}$ e a faixa de linearidade de 0 a $400 \mu \mathrm{g} . \mathrm{L}^{-1}$.

\section{Lançamento do traçador gasoso}

Diferentemente do traçador conservativo, a injeção do traçador gasoso é feita por um período de tempo maior. No entanto, ocorreu no mesmo instante de tempo que o traçador conservativo. Essa metodologia de injeção foi enunciada por Yotsukura et al. (1983), que propuseram a injeção contínua (vazão constante de gás traçador) por um período de tempo, de modo a proporcionar um estado permanente denominado platô.

A um botijão P13 foi acoplado um regulador de pressão GLP Oxiléo OL-032, no qual se ligou um fluxômetro industrial White Martins calibrado para controlar a saída da mistura gasosa. Em uma das extremidades de uma mangueira de borracha de 0,5" de diâmetro, com 14 m de comprimento, conectou-se a saída do fluxômetro.
$\mathrm{Na}$ outra extremidade conectou-se um difusor de ar do tipo retangular, da marca Oxyflex com 160 mm de largura e 660 mm de comprimento. O difusor possuía uma membrana de borracha EPDM e área de aeração de $1.000 \mathrm{~cm}^{2}$.

O difusor foi fixado no fundo do ponto central da seção transversal do curso de água. O lançamento do traçador gasoso ocorreu no mesmo instante do lançamento do traçador conservativo.

A vazão de gás traçador foi estabelecida de forma a garantir a quantificação analítica do traçador na seção mais a jusante. Uma estimativa de vazão foi apresentada por Rathbun e Grand (1978). A vazão de gás necessária para produzir um platô com concentração de $1 \mu \mathrm{g}$. $\mathrm{L}^{-1}$, relacionando-se a eficiência de transferência de massa do difusor, a vazão do curso de água e a dessorção de primeira ordem do propano. Para as condições de $21^{\circ} \mathrm{C}$ e 1 atm, a vazão de gás obtido pela expressão apresentada na Equação 9.

$q_{p}=\frac{118,2 \cdot\left(2 m \cdot e^{T p \cdot K p}\right)}{\varepsilon}$

Onde:

$q_{p}$ : vazão do gás constante injetada (L. $\left.\mathrm{min}^{-1}\right)$;

Qm: vazão máxima na seção mais a jusante $\left(\mathrm{m}^{3} \cdot \mathrm{s}^{-1}\right)$;

Tp: tempo de ocorrência do pico de concentração (h);

Kp: coeficiente de dessorção do gás traçador $\left(\mathrm{h}^{-1}\right)$;

$\boldsymbol{\varepsilon}$ : eficiência da transferência de massa.

A estimativa do coeficiente de dessorção é necessário para o cálculo da vazão do gás traçador. O modelo de Tsivoglou e Neal (1976) transformado para desorção de propano foi utilizado para cursos de água com declividades maiores que $0,003 \mathrm{~m} \cdot \mathrm{m}^{-1}$. Ele é expresso conforme apresentado na Equação 10.

$K_{p}=0,002 \cdot \frac{\Delta H}{T_{p}}$

Onde:

$\Delta H$ : diferença de cota entre os pontos de montante e jusante (m); $T_{p}$ : tempo de ocorrência do pico de concentração (min); $K_{p}$ : coeficiente de dessorção do gás traçador $\left(h^{-1}\right)$.

O modelo de Owens, Edwards e Gibbs (1964) transformado para dessorção de propano foi utilizado para cursos de água com declividades menores que 0,003 m.m-1. Ele é descrito na Equação 11 .

$K_{p}=0,651 \cdot \frac{(v \cdot 3,281)^{0,67}}{d^{1,85}}$

Onde:

$K_{p}$ : coeficiente de dessorção do gás traçador $\left(h^{-1}\right)$;

$v$ : velocidade média $\left(\mathrm{m} . \mathrm{s}^{-1}\right)$;

$\mathrm{d}$ : profundidade média (m). 
O tempo mínimo de injeção constante é o tempo necessário para a produção de um platô. Para se calcular o tempo mínimo de injeção utilizou-se a abordagem de Taylor, James Junior, Helinsky (1984). A aresta comprimento do triângulo escaleno dos autores é uma estimativa do tempo de residência do traçador conservativo lançado pontualmente. Como a injeção do traçador gasoso é feita no mesmo instante do lançamento do traçador conservativo, o tempo mínimo de residência do platô deve ser igual ao tempo de residência do traçador conservativo. O cálculo do tempo mínimo de injeção do traçador gasoso é obtido pela Equação 12.

$T_{i}=3,1 \cdot T_{p}^{0,67}$

Onde:

$\mathrm{T}_{\mathrm{i}}$ : tempo mínimo de injeção (min);

$\mathrm{T}_{\mathrm{p}}$ : tempo de viagem do pico (min).

O tempo total de injeção foi calculado considerado o tempo mínimo de injeção somado ao tempo de coleta. Como o tempo de amostragem foi de 25 minutos, então o tempo total de injeção para cada experimento foi dado por $T_{i}+25$ minutos. Para cada seção foram coletadas cinco amostras. O intervalo de tempo entre as coletas foi de cinco minutos.

A amostragem foi feita no centro do escoamento adotando o método de coleta da pipeta invertida de Formentini (2010). Para coletar a amostra mergulhou-se uma pipeta volumétrica de $15 \mathrm{~mL}$ de forma invertida (com o orifício maior para baixo), numa inclinação de $45^{\circ}$ na direção do escoamento. A amostra completa a pipeta com pouca turbulência, mantendo-se preservadas as características da mesma. Enchida a pipeta, bloqueia-se a parte inferior com o dedo indicador e retira-se o excesso de amostra considerando-se o menisco de calibração. A amostra foi escoada dentro de tubos de vidro âmbar tipo headspace (específicos para a técnica), com volume de $20 \mathrm{~mL}$, e selada com septos de silicone e anéis de alumínio. Uma vez violados, os selos não são reaproveitados. A profundidade de coleta foi de $30 \mathrm{~cm}$ da superfície. Uma vez dentro do frasco, as amostras foram imediatamente refrigeradas em sistema isolado contendo banho de gelo. Ao término dos ensaios, as amostras foram levadas ao laboratório de instrumentação analítica para quantificação do gás propano

\section{Análise do propano}

A determinação da concentração de propano foi feita através de um cromatógrafo gasoso Shimadzu ${ }^{\circledR} 14 \mathrm{~B}$ com detector de ionização de chama. Utilizou-se gás hélio aquecido a $200^{\circ} \mathrm{C}$ como fase móvel, numa vazão de $15 \mathrm{~mL} \cdot \mathrm{min}^{-1}$. A temperatura do detector foi configurada para $230^{\circ} \mathrm{C}$ e a do injetor ajustada para $230^{\circ} \mathrm{C}$. Não foi utilizada curva de aquecimento. Dessa forma, toda a corrida cromatográfica ocorreu na temperatura de $200^{\circ} \mathrm{C}$. Definiu-se 7 min como o tempo de corrida. Configurou-se uma rampa de aquecimento de $24^{\circ} \mathrm{C}$ por minuto até $250^{\circ} \mathrm{C}$ para retirar eventuais impurezas, após a corrida.

$\mathrm{O}$ equipamento foi equipado com uma coluna aberta de camada porosa, com área de $750 \mathrm{~m}^{2} \cdot \mathrm{g}^{-1}$. A coluna Carboxen-1006 PLOT $\left(\right.$ Supelco ${ }^{\circledR}$ ), com $30 \mathrm{~m}$ de comprimento e 0,32 mm de diâmetro. Ela é composta por sílica fundida e indicada para rápida separação de hidrocarbonetos leves $\left(C_{1}, C_{2}\right.$ e $\left.C_{3}\right)$.

A calibração do cromatógrafo ocorreu através do preparo de uma solução concentrada de gás propano em água. Para isso utilizou-se um padrão de gás propano da marca Scott ${ }^{\circledR}$. A metodologia utilizada para o preparo das soluções concentradas e padrões foi baseada nas descrições de Barbosa Junior (1997). Em um frasco de vidro para DBO, com volume de $300 \mathrm{~mL}$, adicionou-se $75 \mathrm{~mL}$ de gás propano sob pressão ambiente e a outra parte foi ocupada com água. Isso foi feito dentro de um aquário cheio de água, onde no frasco inicialmente cheio de água, foi borbulhado gás propano até que ocupasse o volume de $75 \mathrm{~mL}$. O procedimento foi feito com ajuste fino de vazão para permitir a lenta expulsão do líquido até se atingir a proporção de volumes desejada. O frasco foi selado e, logo em seguida, submetido à vigorosa agitação por exatamente $10 \mathrm{~min}$. Terminado o procedimento anterior, a mistura produzida foi colocada em repouso por outros $10 \mathrm{~min}$, mas com o frasco aberto para a atmosfera. Isso permite o escape da porção do gás não dissolvida. A concentração da solução concentrada foi estimada com base nas leis de Dalton e Henry, conforme a Equação 13.

$C_{p}=\frac{P_{a t m}}{16+\frac{0,08205 \cdot T}{44} \cdot \frac{V_{L F}}{V_{G F}}}$

Onde:

$C_{p}$ : concentração do gás propano dissolvido $\left(\mathrm{g} . \mathrm{L}^{-1}\right)$;

$P_{a t m}:$ pressão atmosférica local (atm);

$T$ : temperatura da solução $(K)$;

$\mathrm{V}_{\mathrm{LF}}$ : volume da porção líquida no frasco $(L)$;

$\mathrm{V}_{\mathrm{GF}}$ : volume da porção gasosa no frasco $(L)$.

Preparada a solução concentrada, fez-se a diluição da mesma para produzir a solução de trabalho. A partir desta, preparou-se padrões nas concentrações de 0,5 a 300 g.L-1. Foram produzidas três curvas de calibração, uma para baixas concentrações $\left(R^{2}=0,996\right)$, outra para médias concentrações $\left(R^{2}=0,998\right)$ e uma última, para altas concentrações $\left(R^{2}=0,992\right)$.

A quantificação da concentração de propano nas amostras foi conduzida através da técnica do headspace, utilizando como base o método empregado por Barbosa Junior (1989). Antes da abertura das amostras, submeteram-se os frascos contendo o analito a um banho termostatizado. Como as amostras foram acondicionadas na temperatura de $4^{\circ} \mathrm{C}$ foi necessário elevar e padronizar a temperatura da amostra. A temperatura do banho foi ajustada para 
$40^{\circ} \mathrm{C}$. Padronizada a temperatura, iniciou-se o procedimento de extração e análise do propano. Após a retirada de cada amostra do banho termostatizado, agitou-se vigorosamente por 30 s. Em seguida, deixou-se a mesma em repouso por outros 30 s. Então, rompeu-se o lacre do frasco retirando-se uma alíquota de $250 \mu \mathrm{L}$ da fase gasosa. A retirada da amostra foi feita utilizando uma seringa gastight. Em seguida, injetou-se a alíquota no cromatógrafo gasoso previamente calibrado. A quantificação foi feita através da interpolação da área do pico versus concentração, mediante equação da reta.

\section{Eficiência da injeção do gás propano presente no GLP}

A eficiência da injeção do gás propano $\left(\boldsymbol{\varepsilon}_{i}\right)$ foi calculada através da Equação 14.

$\varepsilon_{\mathrm{i}}=\frac{m_{a}}{m_{i}} \cdot 100$

Equação 14

O valor de $m_{a}$ foi estimado a partir da medida da taxa de transporte de massa do propano na seção de amostragem, acrescendo-se a esse o valor perdido pela dessorção que ocorre no trecho de mistura. O valor de $m_{i}$ foi determinado através da diferença de massa do botijão entre o início e o fim de cada experimento. Reescrevendo-se a Equação 14 obtêm-se a expressão apresentada na Equação 15

$\boldsymbol{\varepsilon}_{i}=\frac{\left(\overline{\mathcal{C}}_{m} Q_{m}\right)^{K_{t} \cdot e^{\bar{t}}}}{m_{i} / t}$

Equação 15

Onde:

t: tempo médio de passagem do gás propano na seção montante;

$\bar{C}_{m}$ : concentração média na seção de amostragem ( $\mu$ g. $\left.L^{-1}\right)$;

$\mathrm{Q}_{m}$ : vazão do corpo de água na seção montante $\left(\mathrm{m}^{3} \cdot \mathrm{s}^{-1}\right)$;

$\mathrm{K}_{t}$ : coeficiente de dessorção do gás propano $\left(\mathrm{dia}^{-1}\right)$; $\mathrm{m}_{i}:$ massa injetada $(g)$;

t: tempo de injeção total do traçador gasoso $(h)$.

\section{Precisão da técnica}

A avaliação da precisão da técnica foi feita levando em consideração a estabilidade dos platôs produzidos. Quanto menor é a variação dos resultados maior é a estabilidade dos mesmos. Utilizou-se o coeficiente de variação (CV\%) por ser uma ferramenta muito útil para comparar a estabilidade dos platôs produzidos em diferentes trabalhos. Compararam-se os CV\% dos platôs obtidos nesta técnica com os obtidos através da técnica original (aquela que utiliza propano de elevado grau de pureza). Os CV\% foram determinados através da Equação 16.

$C V \%=\frac{D P}{C M D} \cdot 100$

Equação 16

Onde:

DP: desvio padrão amostral;

CMD: concentração média determinada.

\section{Resultados e discussão}

\section{Percentual de propano no GLP}

A utilização do GLP como fonte de gás propano utiliza maior volume de gás injetado, pois é necessário compensar a vazão para o percentual de outros constituintes (volume/volume) presente na mistura. Para a execução dos 15 ensaios, foram utilizados 6 botijões P13.

A Figura 1A mostra o cromatograma obtido pela análise do headspace de uma amostra de água onde foi injetado propano de pureza 99,5\% e a Figura 1B mostra o cromatograma obtido pela análise do headspace de uma amostra de água onde foi injetado GLP. Nota-se que o tempo de retenção médio do gás propano foi de 5,33 minutos.

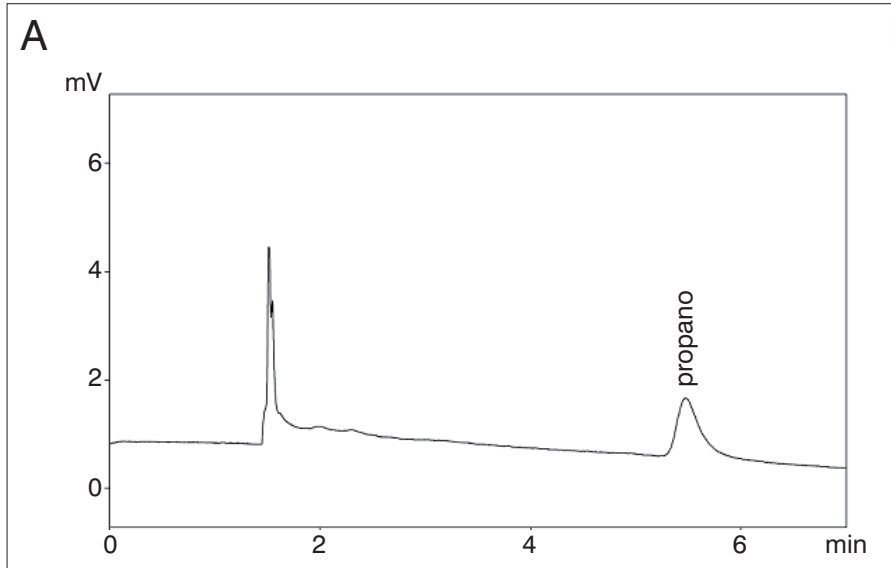

B

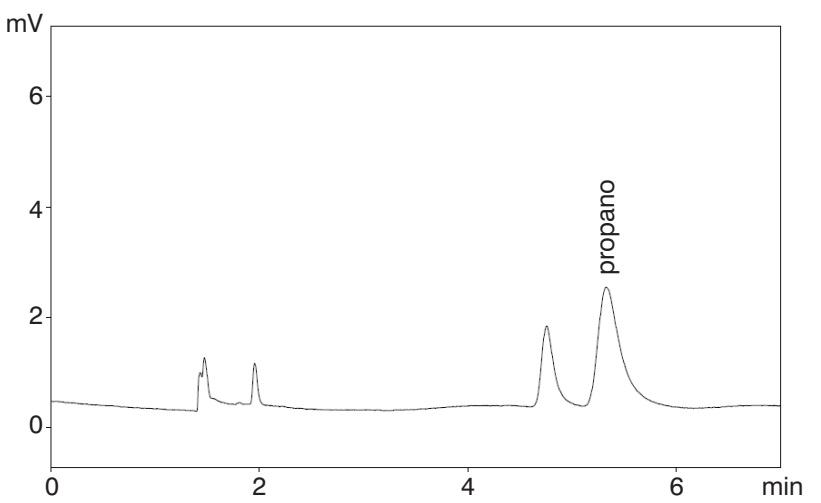

Figura 1 - Cromatograma obtido pela análise do headspace de uma amostra de água onde foi injetado propano 99,5\% de pureza (A) e GLP (B). 


\section{Eficiência na transferência de gás propano}

Assim como a maioria dos hidrocarbonetos gasosos, o propano apresenta baixa solubilidade. Por esse motivo deve-se planejar muito bem a forma de transferi-lo para o meio aquoso. Testes realizados por pesquisadores indicaram que entre 5 e 20\% do gás injetado é realmente absorvido. O excesso escapa no momento da injeção ou a uma curta distância a jusante do ponto de injeção (KILPATRICK et al., 1989).

Observou-se que a eficiência de injeção de gás propano é afetada pela relação largura/profundidade e pela vazão de gás injetado. Quanto maior foi a relação largura/profundidade, menor foi a eficiência da injeção obtida, e quanto maior a vazão de injeção, menor foi a eficiência da mesma (mantendo-se constante a área de difusão). Isso é justificado pela formação de bolhas maiores que se desprendem mais rapidamente do meio aquático, motivo pelo qual Barbosa Junior (1997) desenvolveu um sistema para pré-dissolver o gás traçador. Esse procedimento possibilitou obter uma eficiência média de $23,71 \%$. Formentini (2010), utilizando um difusor projetado a partir de uma mangueira de silicone, apresentou eficiência média de $3,4 \%$ na injeção do gás traçador. Neste trabalho, sem utilizar aparato sofisticado para pré-dissolução, obteve-se uma eficiência média de $3,458 \%$. Os valores das eficiências obtidos em todos os ensaios são apresentados na Tabela 1.

Na Figura 2 são apresentadas as curvas de evoluções temporais das concentrações da rodamina WT e do gás propano obtidas em dois ensaios, nas seções de medições. Nota-se que os platôs de propano ocorreram após a passagem do traçador. Além disto, verifica-se através da comparação matemática das áreas, ou por uma simples inspeção visual, que a qualidade da mistura melhora com o afastamento da seção de injeção. Mesmo não apresentando o fisicamente proposto - comportamento gaussiano da nuvem, pela razoável uniformidade das áreas, pode-se supor que a mistura foi praticamente atingida nos ensaios.

\section{Injeção com sistema banho-maria}

Durante os ensaios percebeu-se que o botijão P13 sofria diminuição de temperatura, o que resultava na redução da pressão e da vazão do gás, prejudicando a formação do platô. Na Figura 3 são apresentados os resultados estatísticos obtidos com as amostras coletadas

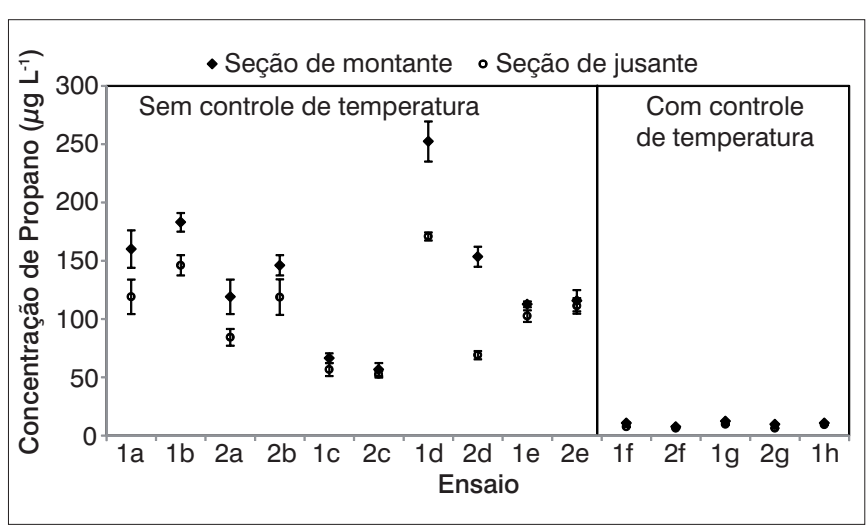

Figura 3 - Concentrações médias e desvios padrões de propano $\left(\mu \mathrm{g} \cdot \mathrm{L}^{-1}\right)$ na amostra de água durante o platô.

Tabela 1 - Eficiência da injeção do gás propano.

\begin{tabular}{|c|c|c|c|c|c|c|c|c|c|c|c|c|c|c|c|}
\hline Ensaio & $1 a$ & $1 b$ & $2 a$ & $2 b$ & $1 c$ & $2 c$ & $1 d$ & $2 d$ & $1 e$ & $2 e$ & $1 f$ & $2 f$ & $1 \mathrm{~g}$ & $2 g$ & $1 \mathrm{~h}$ \\
\hline Q & 0,063 & 0,067 & 0,074 & 0,076 & 0,225 & 0,226 & 0,019 & 0,022 & 0,074 & 0,073 & 4,22 & 4,485 & 0,873 & 0,952 & 0,4456 \\
\hline$q$ & 5 & 5 & 5 & 5 & 5 & 5 & 5 & 5 & 5 & 5 & 30 & 30 & 15 & 15 & 5 \\
\hline$\varepsilon$ & - & - & 4,761 & 4,761 & 5,856 & 5,856 & 2,797 & 4,937 & 4,498 & 6,232 & 0,76 & 0,76 & 0,997 & 0,997 & 2,096 \\
\hline
\end{tabular}

Q: vazão do corpo de água no trecho $\left(\mathrm{m}^{3} \cdot \mathrm{s}^{-1}\right)$; q: vazão de gás GLP injetado (L.min $\left.{ }^{-1}\right)$; $\varepsilon$ : percentual de eficiência obtida na injeção o gás (\%).

A

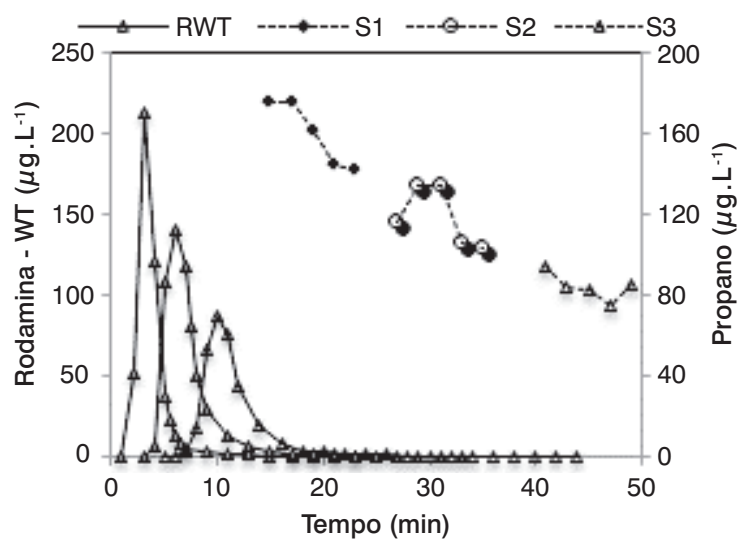

B

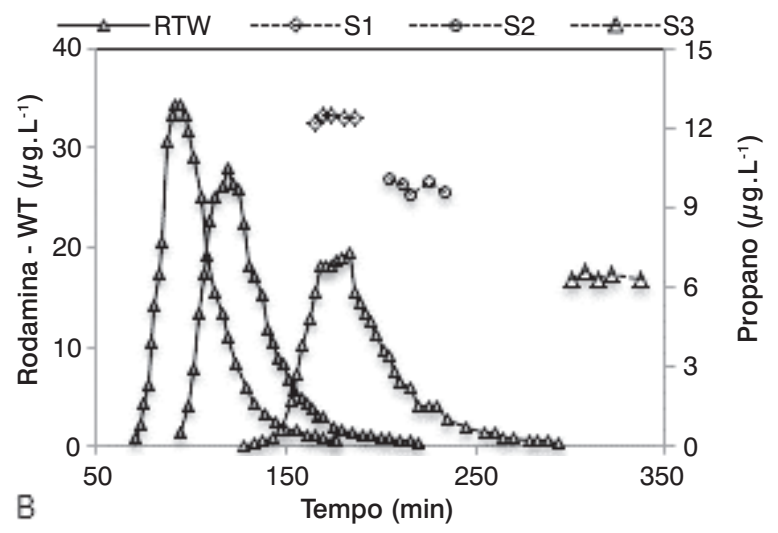

Figura 2 - Concentrações da rodamina WT e do propano, nos ensaios 1a e 2a (A) e $1 \mathrm{~g}$ e $2 \mathrm{~g}(\mathrm{~B})$. 
no momento de ocorrência do platô, tanto nas seções montantes, quanto jusantes. Os resultados mostram grandes desvios padrões. Kilpatrick et al. (1989) já haviam destacado problemas na injeção do traçador gasoso, provenientes do resfriamento do botijão de propano. Salientaram ainda que não é recomendado utilizar nos experimentos botijões que estiverem preenchidos com menos de $20 \%$ da sua capacidade. Sua justificativa está no fato de ocorrer diminuição da área superficial de troca térmica que prejudicaria o experimento.

Mesmo seguindo a recomendação de utilizar no máximo 80\% da capacidade do botijão, os resultados não foram muito satisfatórios, motivo pelo qual se desenvolveu um sistema de controle de temperatura (banho-maria). O botijão foi colocado dentro de um tanque metálico com água e aquecido com uma chama de GLP, até alcançar a temperatura de cerca de $50^{\circ} \mathrm{C}$. A solução encontrada e testada possibilitou o aquecimento controlado do botijão, evitando que houvesse a redução da pressão e vazão. Na Figura 3 são apresentados os resultados estatísticos obtidos com as amostras coletadas no platô, utilizando o sistema de controle de temperatura.

A análise dos dados obtidos mostra que os desvios padrão são menores com o sistema de controle da temperatura. Este sistema mostrou-se uma excelente solução para garantir a estabilidade do platô. A garantia de estabilidade do platô resulta em ganho de precisão da técnica.

$\mathrm{O}$ aquecimento do botijão até a temperatura de $50^{\circ} \mathrm{C}$ não oferece risco de segurança, pois o mesmo contém um plugue fusível como válvula de segurança. Se o aquecimento atingisse a temperatura de $70^{\circ} \mathrm{C}$, o plugue fusível, formado principalmente por uma liga de bismuto e estanho, sofreria fusão, escorrendo para dentro do botijão. Isso possibilitaria a saída do gás e evitaria a possibilidade de explosão do botijão.

\section{Precisão da técnica}

O controle da temperatura através do sistema banho-maria tornou a técnica muito mais precisa do que aquela que utiliza o gás propano de pureza 99,5\%. O coeficiente de variação possibilitou a comparação da precisão dos resultados de diferentes trabalhos. Na Figura 4 são apresentadas as comparações da precisão da técnica sem o sistema de banho-maria e com o sistema de banhomaria. O coeficiente de variação se mostrou menor para a injeção utilizando o sistema de banho-maria. Quanto mais perto do ponto central estão os coeficientes de variação melhor é a precisão da técnica, pois a dispersão dos valores de concentração de propano estaria tendendo a zero.

O coeficiente de variação médio para a técnica de injeção utilizando o sistema de banho-maria foi de 3,3\%, enquanto para a técnica de injeção sem o sistema banho-maria foi de 7,2\%. Este trabalho utilizou procedimentos metodológicos (planejamento experimental, lançamento do traçador conservativo, análise do traçador conservativo

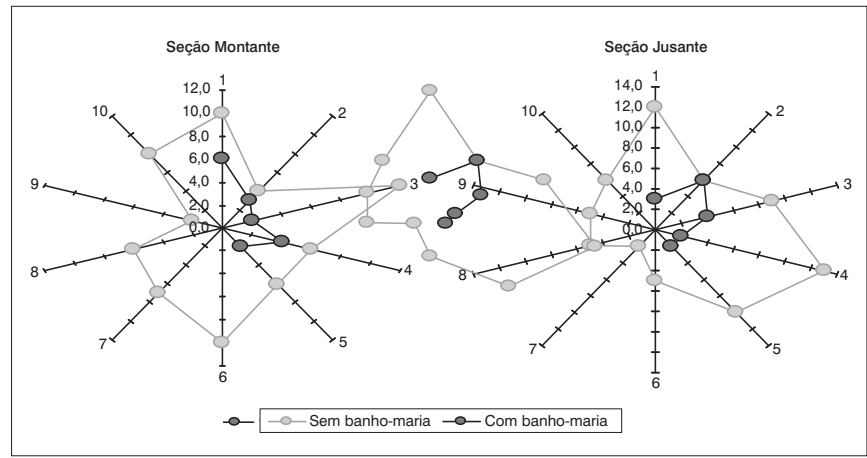

Figura 4 - Coeficiente de variação médio para os sistemas com e sem controle de temperatura.

e análise do propano) iguais ao de Formentini (2010) que utilizou gás propano de pureza 99,5\%. As variações estiveram no sistema de injeção do gás traçador e nos frascos para armazenamento das amostras para a técnica de headspace. O coeficiente de variação médio dos experimentos de Formentini (2010) foi de 10,5\%. Já Barbosa Junior (1997), utilizando seu sistema de pré-dissolução do gás propano, atingiram o coeficiente de variação médio de 3,9\%. Isso demonstra que a precisão desse método, somada à utilização do sistema de controle de temperatura, gera resultados equiparáveis e até superiores aos métodos que utilizam propano de elevado grau de pureza.

\section{Custo experimental}

O fator financeiro é o principal fator limitante para o amplo uso da técnica dos traçadores gasosos no Brasil, motivo pelo qual soluções alternativas a técnica ainda são procuradas por pesquisadores brasileiros. Este é o caso do trabalho de Costa (1999), que aperfeiçoou o método da sonda solúvel (estimativa indireta de $\mathrm{K}_{2}$ ). Segundo a autora, esse seria um método mais simples e econômico quando comparado com as demais técnicas. Mostrando o elevando custo da técnica dos traçadores gasosos utilizando propano puro, destaca-se o trabalho de Formentini (2010), que atingiu o custo médio experimental de $\mathrm{R} \$ 900,00$. Esse valor considera apenas o material consumido em um experimento, e para reduzir esse custo, Formentini (2010) consideraram a hipótese de melhorar o sistema de injeção de gás propano para aumentar o rendimento da transferência de massa. Nesse sentido, Barbosa Junior (1997) otimizou o processo de transferência de massa, atingindo rendimentos superiores aos registrados por Kilpatrick et al. (1989).

No Brasil, o custo de um cilindro de gás propano de aproximadamente $45 \mathrm{~kg}$ (100 libras), com grau de pureza de 99,5\%, é de $\mathrm{R} \$$ 8.000,00 (mês de referência 07/2010), enquanto, o custo de um botijão P13 (GLP) é em média de R\$42,00. O sistema de injeção desenvolvido neste trabalho é mais elaborado que o de Formentini (2010), porém, muito mais simples que o de Barbosa Junior (1997). O custo experimental da técnica com GLP 
estabilizado termicamente não sofreria grandes reduções pela melhoria do sistema de injeção, motivo pelo qual não se focou no aperfeiçoamento do mesmo. Para a execução dessa técnica gastouse com gás GLP o valor médio de R \$ 16,80 por experimento. Fazendo-se uma estimativa do valor médio gasto por experimento, caso fosse feita a substituição do GLP por gás propano 99,5\%, o valor seria de R\$452,98. A utilização do GLP como fonte do gás traçador propano possibilitou a redução do custo em material de consumo de aproximadamente 27 vezes, resultando numa economia de $\mathrm{R} \$ 436,18$ por experimento.

\section{Determinação do coeficiente de reaeração}

A técnica do traçador gasoso GLP foi testada em corpos de água e trechos com características hidráulicas diferentes. Os coeficientes de reaeração foram determinados para cada trecho situados entre as seções de coleta de amostras de montante e de jusante. Os valores determinados foram convertidos para a temperatura de $20^{\circ} \mathrm{C}$. Os coeficientes de reaeração são apresentados na Tabela 2. Além dos coeficientes de reaeração é possível observar as características hidrodinâmicas de cada trecho onde a técnica foi testada.

Constata-se a forte relação entre os dados hidráulicos e os coeficientes de reaeração obtidos, conforme é apresentado na Figura 5. Tal como em Melching e Flores (1999), Jha, Ojha e Bathia (2001; 2004), as características hidrodinâmicas dos cursos de água representaram fatores fortemente relacionados com o coeficiente de reaeração. Os ensaios realizados no ribeirão Concórdia (ensaios a, b e c), e nos arroios Dona Lúcia (ensaio d) e Braço do Concórdia (ensaio e) apresentaram trechos com altas taxas de reaeração. Para os ribeirões Garcia (ensaio f), Velha (ensaio g) e Fortaleza (ensaio h), os valores obtidos para o coeficiente de reaeração foram mais baixos. Nota-se significativa diferença entre as características hidrodinâmicas entre entes dois conjuntos de cursos de água. Enquanto os primeiros apresentaram vazões inferiores a $0,23 \mathrm{~m}^{3} . \mathrm{s}^{-1}$, os segundos apresentam valores superiores a $0,44 \mathrm{~m}^{3} \cdot \mathrm{s}^{-1}$, chegando a $4,485 \mathrm{~m}^{3} \cdot \mathrm{s}^{-1}$. Outras diferenças importantes podem ser visualizadas nos outros parâmetros hidrodinâmicos dos cursos de água.

\section{Conclusões}

Os experimentos mostram que a utilização do GLP como fonte do traçador propano é viável para a determinação do coeficiente de reaeração. No entanto, para a sua utilização é necessário corrigir a injeção da mistura gasosa compensando o percentual de propano presente. Nos botijões P13 utilizados encontrou-se a média de 49\% de gás propano presente na mistura

Como o botijão P13 sofria diminuição de temperatura, o que provocava a redução da precisão dos experimentos, passouse a estabilizar a temperatura do botijão através de um sistema banho-maria $\left(50^{\circ} \mathrm{C}\right)$. O sistema não oferece risco se manuseado com cuidado. A estabilização de temperatura possibilitou ganho de precisão de mais de $50 \%$, pois coeficiente de variação do platô passou de 7,2\% (sem sistema de controle de temperatura) para 3,3\%. O GLP mostrou ser um grande aliado dos ensaios de reaeração, pois além de gerar resultados equiparáveis e até superiores aos da técnica que utiliza o propano de alto grau de pureza, ele é facilmente encontrado.

O GLP ofereceu comodidade durante as experimentações. Os botijões P13 são leves e facilmente transportáveis. Não é necessário instalar nos locais sistemas sofisticados de gás. Todo aparato levado ao campo é facilmente retirado após a experimentação.

A maior contribuição do GLP como fonte do gás traçador propano é sem dúvida a economia gerada. O método do traçador gasoso GLP resultou numa economia de R $\$ 436,18$ por experimento, mostrando que é a determinação de parâmetros de atuação na capacidade de autodepuração dos corpos de água a custo muito mais baixo do que a técnica tradicional, sem perda de confiabilidade dos resultados.

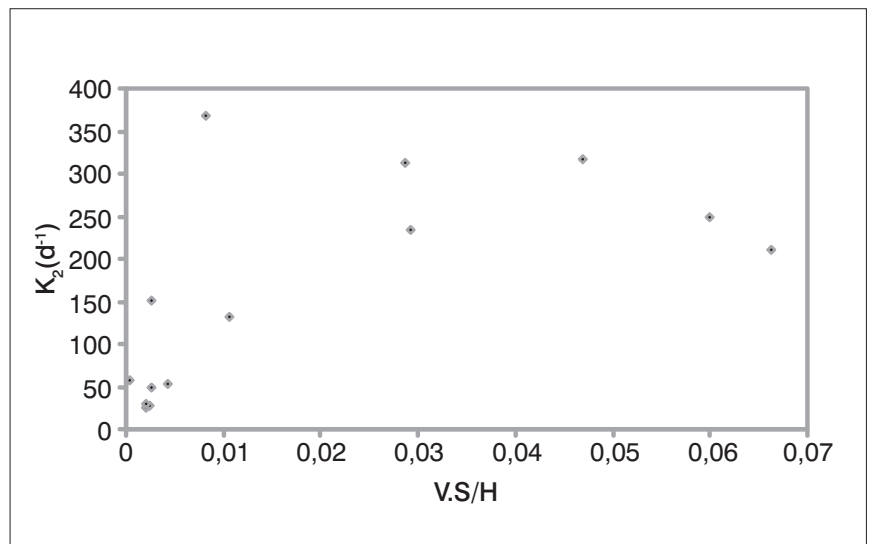

Figura 5 - Relação entre os coeficientes de reaeração e 0 fator V.S/H, onde $V$ é a velocidade média do escoamento $\left(\mathrm{m} \mathrm{S}^{-1}\right)$, S é a declividade do curso de água $\left(\mathrm{m} \cdot \mathrm{m}^{-1}\right)$ e $\mathrm{H}$ é a profundidade media do escoamento no trecho (m).

Tabela 2 - Características hidráulicas dos trechos e coeficientes de reaeração

\begin{tabular}{|c|c|c|c|c|c|c|c|c|c|c|c|c|c|c|c|}
\hline Ensaio & $1 a$ & $1 b$ & $2 a$ & $2 b$ & $1 c$ & $2 c$ & $1 d$ & $2 d$ & $1 e$ & $2 e$ & $1 f$ & $2 f$ & $1 \mathrm{~g}$ & $2 \mathrm{~g}$ & $1 \mathrm{~h}$ \\
\hline Q & 0,063 & 0,074 & 0,067 & 0,076 & 0,225 & 0,226 & 0,019 & 0,022 & 0,075 & 0,073 & 4,22 & 4,485 & 0,873 & 0,952 & 0,445 \\
\hline $\mathrm{H}$ & 0,245 & 0,200 & 0,230 & 0,198 & 0,386 & 0,258 & 0,174 & 0,322 & 0,245 & 0,213 & 0,341 & 0,341 & 0,368 & 0,320 & 0,292 \\
\hline$B$ & 1,50 & 1,80 & 0,80 & 1,05 & 3,60 & 2,15 & 1,17 & 1,57 & 2,12 & 2,15 & 14,61 & 14,61 & 6,95 & 8,35 & 5,25 \\
\hline$k_{2}$ & 313,1 & 250,6 & 317,2 & 211,2 & 152,3 & 133,1 & 234,4 & 367,7 & 59,3 & 27,0 & 54,5 & 25,8 & 49,1 & 27,9 & 30,1 \\
\hline
\end{tabular}

Q: vazão no trecho $\left(\mathrm{m}^{3} \cdot \mathrm{s}^{-1}\right)$; H: profundidade média do escoamento no trecho $(\mathrm{m})$; B: largura média do rio no trecho $(\mathrm{m})$; $\mathrm{K}_{2}$ : coeficiente de reaeração $($ dia-1 $)$. 


\section{Referências}

AMBROSE, R.B.; WOOL, T.A.; BARNWELL, T.O. (2009) Development of water quality modeling in the United States. Environmental Engineering Research, v. 14, n. 4, p. 200-10.

BARBOSA JUNIOR, A.R. (1989) Desenvolvimento de metodologia para a determinação do coeficiente de reaeração dos escoamentos naturais de água com o emprego de traçador gasoso. 187 f. Dissertação (Mestrado) - Escola de Engenharia de São Carlos da Universidade de São Paulo, São Carlos.

BARBOSA JUNIOR, A.R. (1997) Uso de traçadores na determinação de parâmetros de qualidade em escoamento naturais da água. 456 f. Tese (Doutorado) - Escola de Engenharia de São Carlos da Universidade de São Paulo, São Carlos.

BONING, C.W. (1973) Index to time-of-travel studies of the U.S. Geological Survey. U.S. Geological Survey Water-Resources Investigations 34-73, 71 p.

COSTA, O.S. (1999) Contribuição à metodologia para determinação indireta do coeficiente de reaeração dos escoamentos naturais da água com emprego do método da sonda solúvel. 187 f. Tese (Doutorado) Escola de Engenharia de São Carlos da Universidade de São Paulo, São Carlos.

COX, B.A. (2003) A review of dissolved oxygen modelling techniques for lowland rivers. The Science of the Total Environment, v. 314-316, p. 303-34.

FAN, C.; KO, C.H.; WANG, W.S. (2009) An innovative modeling approach using Qual2K and HEC-RAS integration to assess the impact of tidal effect on River Water quality simulation. Journal of Environmental Management. v. 90, n. 5, p.1824-32.

FISCHER, H.B; LIST, E.J; KOH, R.C.Y; IMBERGER, J; BROOKS, N.H. (1979) Mixing in inland and coastal waters. San Diego. Academic Press.

FORMENTINI, T.A. (2010) Coeficientes de desoxigenação e de reaeração superficial em trechos do rio Vacacaí Mirim. 90 f. Dissertação (Mestrado em Engenharia Civil) - Universidade Federal de Santa Maria, Santa Maria.

GAMESON, A.L.H.; TRUESDALE, G.A.; DOWNING, A.L. (1955) Reaeration studies in lakeland beck. Journal of Institution of Water Engineers. v. 9, p. 57-94

GLEIZER, S. (1992) Determinação experimental do coeficiente de reaeração em rios com o uso de traçador inerte gasoso. Dissertação (Mestrado em Engenharia Civil) - Universidade Federal do Rio de Janeiro, Rio de Janeiro.

JHA, R; OJHA, C.S.P; BHATIA, K.K.S. (2001) Refinement of predictive reaeration equations for a typical Indian river. Hydrological Processes, v. 15, p. $1047-1060$.

JHA, R; OJHA, C.S.P; BHATIA, K.K.S. (2004) A supplementary approach for estimating reaeration rate coefficients. Hydrological Processes, v. 18, p. 65-79.

KILPATRICK, F.A.; RATHBUN, R.E.; YOTSUKURA, N.; PARKER, G.W.; DELONG, L.L. (1989) Determination of stream reaeration coefficients by use of tracers. Denver, United States Geological Survey, 52 p.
KILPATRICK, F.A; COBB, E.D. (1985) Measurement of discharge using tracers. Alexandria. U.S. Geological Survey.

LICO, M.S; TAYLOR, R.L. (2000) Reaeration-rate coefficients for two reaches on the Truckee River downstream from Reno, Nevada, 1999 Carson City. U.S. Geological Survey.

LIQUIGÁS DISTRIBUIDORA S.A.(2011). Ficha de informações de segurança de produto químico 9000113. Disponível em: http://mww.liquigas. com.br/wps/wcm/connect/a94a95004640d0be8ce7cddd2947447b/ $\mathrm{FISPQ}+\mathrm{GLP}+$ rev+01-11.pdf?MOD=AJPERES. Acesso em: maio 2012

MELCHING, C.S.; FLORES, H.E. (1999) Reaeration equations derived from U.S. Geological Survey Database. Journal of Environmental Engineering, v. 125, p. 407-14

OWENS, M.; EDWARDS, R.W.; GIBBS, J.W. (1964) Some reaeration studies in streams. International Journal of Air and Water Pollution, v. 8 n. 8/9, p. 469-86.

PARKER, G.W.; GAY, F.B. (1987) A procedure for estimating reaeration coefficients for Massachusetts streams. U.S. Geological Survey WaterResources Investigations Report 86-4111, 38 p.

RATHBUN, R.E; GRAND, R.S. (1978) Comparison of the radioactive and modified techniques for measurement of stream reaeration coefficients. U.S. Geological Survey Water Resources Division, 57 p.

STREETER, H.W.; PHELPS, E.B. (1925) A study of the pollution and natural purification of the Ohio River. U.S. Public Health Service, Public Health Bull. 146. $75 \mathrm{p}$

TAYLOR, K.R.; JAMES JUNIOR, R.W.; HELINSKY, B.M. (1984) Traveltime and dispersion in the Potomac River, Cumberland, Maryland, to Washington, D.C. U.S. Geological Survey Open-File Report 83-861, 55 p.

TSIVOGLOU, E.C.; NEAL, L.A. (1976) Tracer measurement of reaeration, Part 3, Predicting the reaeration capacity of inland streams. Journal of the Water Pollution Control Federation, v. 48, n. 12, p. 2669-89.

TSIVOGLOU, E.C.; WALLACE, J.R. (1972) Characterizing stream reaeration capacity. U.S. Environmental Protection Agency Report. EPAR3-72-012.

U.S. ENVIRONMENTAL PROTECTION AGENCY (USEPA). Office of Drinking Water. (1989) Guidance Manual for Compliance with the Filtration and Disinfection Requirements for Public Water Systems Using Surface Water Sources. Report n. EPA 570/9-89-018, Appendix C-9.

YOTSUKURA, N.; STEDFAST, D.A.; DRAPER, R.E.; BRUTSAERT, W.H. (1983) An assessment of steady-state propane-gas tracer method for reaeration coefficients - Cowaselon Creek. New York. U.S. Geological Survey Water-Resources Report 83-4183, 88 p.

YOTSUKURA, N.; STEDFAST, D.A.; JIRKA, G.H. (1984) An assesment of steady-state propane-gas tracer method for determining reaeration coefficients - Chenango River. New York. U.S. Geological Surver WaterResources Investigations Report 84-4368, 69 p. 